\title{
Planeamiento de Escenarios Estratégicos en la Universidad Pública del Perú
}

\author{
Planning of Strategic Scenarios in the \\ Public University of Peru
}

\begin{abstract}
RESUMEN
El planeamiento de escenarios estratégicos en la universidad pública en el Perú a través de la metodología de la prospectiva y las competencias de los recursos humanos, permite potenciar la gestión académica y administrativa de la universidad. La formulación, la implementación y el control del modelo requiere de un liderazgo eficaz del rector y de los miembros del Consejo de Universitario. En estos tiempos es necesario dejar de lado la lucha por los intereses particulares, y centrarse en el bienestar de los estudiantes, docentes y no docentes y en expandir las acciones de la Universidad a otros ámbitos nacionales e internacionales. El modelo de planeamiento de escenarios estratégicos desarrolla las siguientes actividades: la decisión estratégica; identificación de los factores importantes de decisión (FIDE); identificación de las fuerzas impulsoras del entorno (FIE); matriz del impacto e incertidumbre; selección de escenarios; tabla comparativa de escenarios; amenazas y oportunidades por escenarios.

Palabras claves: Planificación de escenarios; factores de decisión importantes; fuerzas impulsoras del medio ambiente.
\end{abstract}

\begin{abstract}
The Planning of strategic scenarios in the Public University in Peru through the prospective methodology and the competences of the human resources allows to enhance the academic and administrative management of the university. The formulation, implementation and control of the model requires effective leadership of the Rector and the Members of the University Council. In these times it is to set aside the fight for private interests, but to focus on the welfare of students, teachers and non-teachers and to expand the actions of the University to other national and international levels. The strategic scenario planning model develops the following activities: The strategic decision; identification of important decision factors (FIDE); identification of the driving forces of the environment (FIE); impact and uncertainty matrix; selection of scenarios; comparative table of scenarios; threats and opportunities by scenarios.
\end{abstract}

Keywords: Scenario planning; important decision factors; driving forces of the environment.

\section{Kennedy Narciso Gómez ${ }^{1}$}

Kennedy_narc1@hotmail.com

\section{José Villacorta Huapaya ${ }^{1}$}

jvillacortah@unmsm.edu.pe

${ }^{1}$ Universidad Nacional Mayor de San Marcos, Facultad de Ciencias Administrativas. Lima, Perú 


\section{INTRODUCCIÓN}

La universidad pública en el Perú, luego de 31 años de vigencia de la Ley Universitaria $\mathrm{N}^{\circ} 23733$ de 1983, tuvo una nueva Ley Universitaria, la Ley $\mathrm{N}^{\circ} 30220$, de fecha 08 de julio de 2014. En su artículo 12 señala la creación de la Superintendencia Nacional de Educación Superior (Sunedu) como Organismo Público Especializado adscrito al Ministerio de Educación cuyo objetivo es elaborar el planeamiento de escenarios estratégicos de la universidad pública en el Perú.

El planeamiento de escenarios estratégicos en la universidad pública no es utilizado en nuestro país, quizás porque se desconoce la importancia y ventajas que generaría este enfoque en la gestión institucional, pues es una herramienta de gestión empresarial, inmersa en el plan estratégico institucional de la universidad pública, relacionada al plan estratégico institucional de la Sunedu para el periodo 2019-2022 y los lineamientos técnicos de planificación del Sistema Nacional de Planeamiento Estratégico (Sinaplan), articulado en el Plan Estratégico Sectorial Multianual de educación (Pesem).

Yori et al. (2011) señalan que «[...] La planificación de escenarios constituye un instrumento para la toma de decisiones que permite manejar y predecir ambientes de rápido cambio social y de interacciones complejas, disminuyendo el nivel de incertidumbre a través de la descripción de futuros alternativos posibles». Ello significa que las decisiones que se toman e implementan serán efectos de interés por ver su valor para la sociedad universitaria en el futuro; por ejemplo, en estos momentos la decisión de obtener el Licenciamiento de la Universidad Nacional Daniel Alcides Carrión de Cerro de Pasco, entonces el estudio del futuro será consecuencia directa de la incertidumbre, ya que el interés por los docentes, no docentes, alumnos y grupo de interés será el interés que tengan de ésta y mayor será su deseo por conocer el futuro del entregable. Si la Sunedu decide otorgarle el Licenciamiento a la mencionada universidad nacional por 15 años entonces habrá generado un impacto positivo; es así como se opera el proceso de planeamiento por escenarios.

Los mencionados autores señalan: «[...] el método de escenarios aplicado a la toma de decisiones institucional, ha sido adoptado de la multinacional Shell Internacional a finales de los años setenta, a través de la adopción de este método en las rutinas de trabajo de gestión, en lugar de la predicción tradicional para diseñar estrategia institucional». Estas buenas prácticas actualmente están siendo usadas en nuestro país con entregables de valor significativo en el nivel de gobierno y nivel local; la universidad pública tiene un reto de hacerlo y obtener resultados significativos en la gestión pública. Finalmente, los autores consideran la utilidad de los escenarios en el contexto de la estrategia y dicen: «[...] la planificación de escenarios constituye una herramienta que permite mantener informado al gerente de los cambios suscitados a nivel de las variables macroeconómicas claves, entre ellas económicas, sociales, políticas, tecnológicas, gubernamentales, competitiva y demográficas, entre otras, que acontece en el entorno las cuales provienen del análisis estratégico».

La teoría de Minztberg (1994) sobre gestión del talento humano dice: «Ningún trabajo es más vital para nuestra sociedad que el de los directivos. El directivo es el que determina si nuestras instituciones sociales nos atienden bien o si desperdician nuestros talentos y recursos [...] La investigación realizada por el Foro de planificación de los EE.UU. descubrió que solo el $25 \%$ de las empresas consideraban que sus procesos de planificación estratégica eran efectivos y los consultores de estrategias de OC\&C observaron que lo más humano que se puede hacer con la mayoría de los procesos de planificación estratégica es acabar con ellos». Vistos los resultados de la forma de pensar de gerentes sobre la planificación de organizaciones norteamericanas, debe llamar la atención y motivar a la reflexión que en las instituciones del Estado se da mucha importancia a formalizar, codificar y poner en práctica las estrategias que la universidad ya tiene, por lo que se ha conceptualizado el planeamiento de escenarios en que se interpreta que la estrategia no puede ser planeada debido a que es una perspectiva y que se ocupa de la síntesis. Asimismo, añade el autor: «La planificación florece en tiempos estables». Hoy en día, debido a la globalización, gran parte de lo que se considera información "dura" a menudo no es nada: por la falacia de "medir lo que es medible", los resultados son limitantes; por ejemplo, la tendencia a favorecer las estrategias de liderazgo en cos- 
tos enfatizando las eficiencias operativas, que son medibles sobre las estrategias de liderazgo de productos, enfatizando el diseño innovador, que tiende a ser menos medible.

Para obtener una comprensión real y útil de la situación competitiva, los datos blandos deben integrarse en el proceso de planificación; si bien los datos duros pueden informar al intelecto, son en gran medida los datos suaves los que generan sabiduría, son indispensables para la síntesis, la clave para elaborar estrategias. La elaboración de estrategia, explica el autor, deriva de la síntesis informal y visionario, en lugar de programado y formalizado, se basa en el pensamiento divergente, la intuición, involucra a una variedad de actores capaces de experimentar y luego integrarse (Minztberg 1994). Ello significa que en la gestión pública universitaria en el Perú, las autoridades toman las decisiones gerenciales considerando el plan estratégico institucional y las normas legales $\mathrm{y}$ administrativas vigentes.

Finalmente, la única manera de avanzar en la gestión universitaria es responder a la siguiente interrogante: ¿en qué medida el planeamiento de escenarios estratégicos en la universidad pública permitirá optimizar la gestión institucional? Si el objetivo es elaborar el planeamiento de escenarios estratégicos para la universidad pública, ello implica cuatro etapas:

1. Identificar las fuerzas impulsoras del entorno.

2. Identificar y desarrollar los factores importantes de decisión (FIDE).

3. Desarrollar la selección de escenarios.

4. Presentar la matriz de amenazas y oportunidades por escenarios.

\section{RESULTADOS}

El gobierno de la universidad pública en el Perú, según la Ley $\mathrm{N}^{\circ} 30220$, Ley Universitaria, en el capítulo VII, artículo $\mathrm{N}^{\circ} 55$, menciona que la universidad es ejercida por «[...] la Asamblea Universitaria; el Consejo Universitario; el Rector; los Consejos de Facultad; los Decanos», y en el artículo 60 señala que «[...] el Rector es el personero y representante legal de la universidad, tiene a su cargo y a dedicación exclusiva, la dirección, conducción y gestión del gobierno universitario en todos su ámbitos, dentro de los límites de la Ley Universitaria y del Estatuto». Creemos con firmeza que las instancias ejercidas en los diversos órganos de gobierno universitario no solo deben ser inteligentes sino requiere de moral, debido a las observaciones y evidencias de la corrupción en la gestión de justicia que atraviesa nuestro país que imita en las instituciones del sector público y que debemos superar. Por otro lado, consideramos que la formulación de programas, planes, proyectos y estrategias generales o por áreas académicas y administrativas son un lugar común, pero los de recursos humanos no están siendo orientadas con un sentido apropiado de las prioridades en las facultades académicas que generan resistencia a los cambios planteados por la Ley $\mathrm{N}^{\circ} 30220$.

A continuación, se desarrollará la información debidamente procesada y sistematizada en conocimientos acerca de las fases de la metodología prospectiva aplicada al planeamiento de escenarios. Véase figura 1, el método prospectivo de la universidad pública.

La universidad pública está obligada a adecuarse a las exigencias de la Nueva Ley Universitaria $\mathrm{N}^{\circ} 30220$. En el capítulo II se menciona a la Sunedu y en el artículo 12 se indica que «La creación de la Superintendencia Nacional de Educación Superior Universitaria (SUNEDU) adscrita al Ministerio de Educación [...] ejerce su jurisdicción a nivel nacional, con su correspondiente estructura orgánica» y en el artículo 13 indica la finalidad de la Sunedu: «Es responsable del Licenciamiento para el servicio educativo superior universitario, entendiéndose el licenciamiento como el procedimiento que tiene como objetivo verificar el cumplimiento de condiciones básicas de calidad para ofrecer el servicio educativo superior universitario y autorizar su funcionamiento [...] La autorización otorgada mediante el Licenciamiento por la SUNEDU es temporal y renovable y tendrá una vigencia mínima de 6 (seis) años».

La Universidad Pública es gestionada de acuerdo a su Estatuto, Manuales, Reglamentos y normas administrativas y legales vigentes; asimismo, formula, implementa y es ,monitoreado a través del planeamiento de escenarios estratégicos institucional (PEEI) y a partir de ello se generarán el planeamiento de escenarios estratégicos de las facultades y divisiones de 

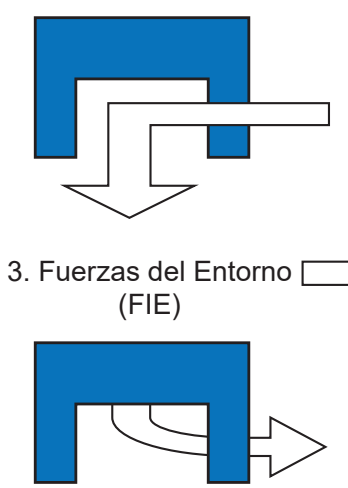

1. Decisión de la Universidad Pública:

$2019-2022$

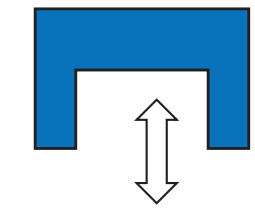

2. Factores Importantes de Decisión (FIDE)

4. Lógica de Escenarios

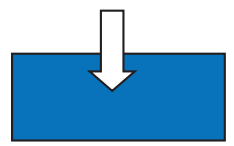

5. Escenarios

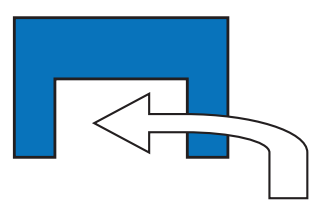

6. Implicancias de la decisión

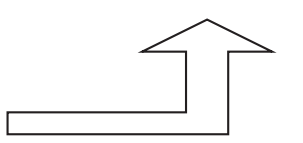

Figura 1. Pasos del método de prospectiva en la universidad. Elaboración propia, 2019.

la estructura orgánica de la universidad para impulsar el resultado de la misión institucional y las estrategias alternativas propuestas; lo descrito nos lleva a plantearnos la siguiente interrogante: ¿Qué estrategia debería implementar la universidad pública para contribuir con la formación académica profesional de calidad? El autor del presente artículo sugiere que el planeamiento de escenarios estratégicos en la universidad pública permitirá lo que la alternativa estratégica genérica de Porter (2004:80) sugiere sobre el liderazgo en costos, a través de la cadena de valor y la manera de asociar estos costos y activos con las actividades de valor.

\section{Fase 1: Enfoque de la decisión de la uni- versidad}

¿Qué estrategias debería implementar la universidad pública 2019-2022 para contribuir con la formación académica profesional de calidad?

El planeamiento de escenarios estratégico de la universidad pública es la estrategia que se debe implementar, y se recomienda el liderazgo en costos, lo que implica brindar sus servicios al menos costo posible.

\section{Fase 2. Identificación de los factores importantes de decisión (FIDE)}

El Planeamiento de Escenarios Estratégicos es la decisión organizacional de la universidad; véase tabla 1.

\section{Fase 3. Identificación de las fuerzas impulsoras del entorno (FIE) y la agrupación de las fuerzas impulsoras del entorno de la universidad pública}

La universidad pública tiene como objetivo preparar y formar hombres en el rigor de la ciencia, tecnología y la humanística; a través de una adecuada gestión de los recurso humanos se propone tener en el corto plazo una infraestructura de primera línea, grato ambiente de estudios, servicios básicos, biblioteca especializada, medios audiovisuales y materiales educativos; además de estructurar y desarrollar la Identificación de los Factores Importantes de Decisión (FIDE) y de agrupar a las Fuerzas Impulsoras del Entorno (FIE). Véase la tabla 2 y la tabla 3.

\section{Fase 4: Lógica de escenarios. Matriz de Impacto-Incertidumbre y Matriz de Lógica de Escenarios}

Se usaron los siguientes escenarios:

- Liberal-Estable.

- Liberal-Inestable.

- Conservador-Estable.

- Conservador-Inestable.

Véase tabla 4, figura 2; tabla 5. 
Planeamiento de Escenarios Estratégicos en la Universidad Pública del Perú

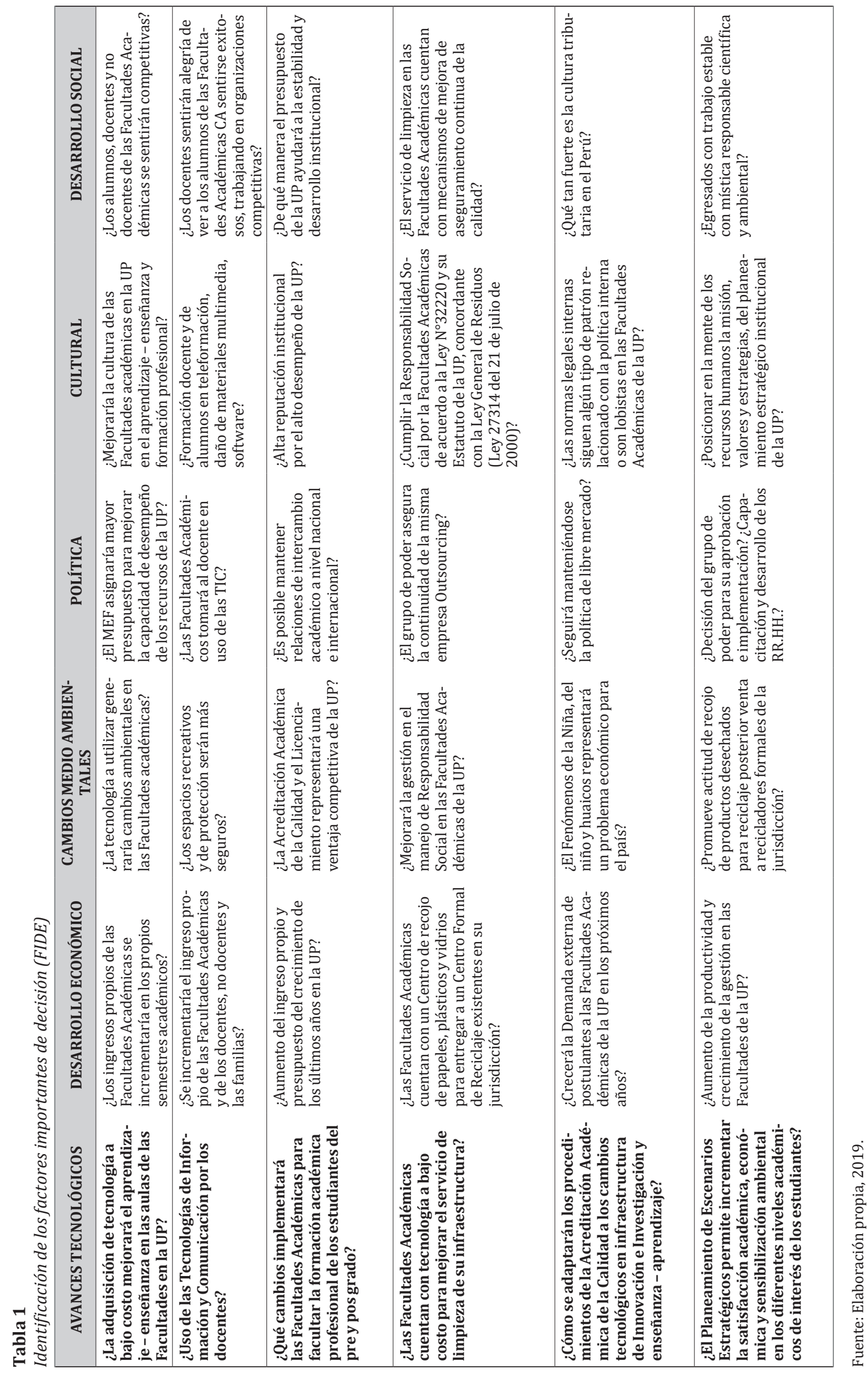


Tabla 2

Identificación de las Fuerzas Impulsoras del Entorno (FIE)

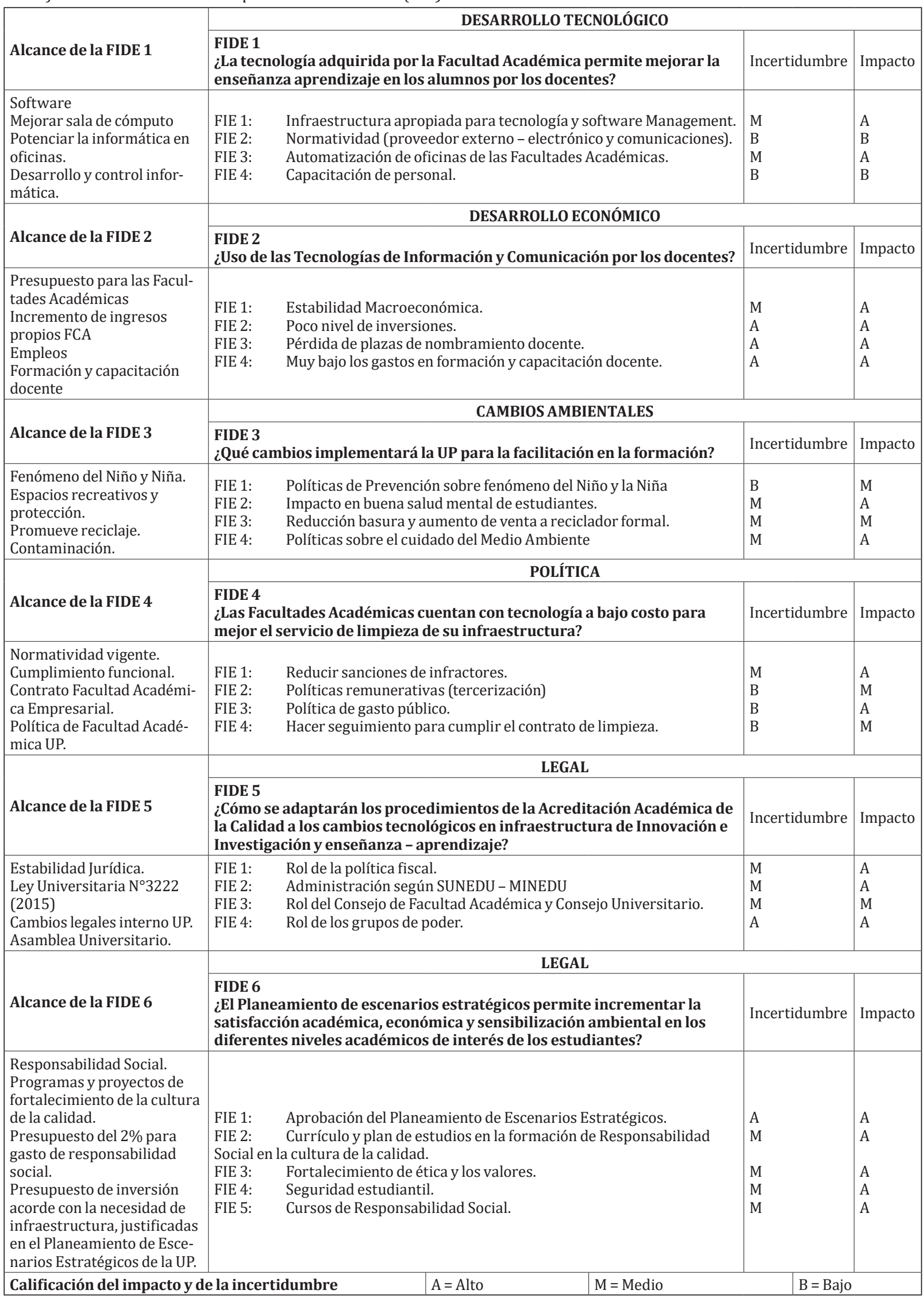

Fuente: Elaboración propia, 2019. 
Tabla 3

Paso 3: Agrupación de las FIE - Universidad Pública

\begin{tabular}{|c|c|c|c|c|c|}
\hline FIDE 1 & FIDE 2 & FIDE 3 & FIDE 4 & FIDE 5 & FIDE 6 \\
\hline $\begin{array}{l}\text { FIE 11: Infraes- } \\
\text { tructura apropiada } \\
\text { para tecnología y } \\
\text { software. }\end{array}$ & $\begin{array}{l}\text { FIE 21: Estabili- } \\
\text { dad Macroeconó- } \\
\text { mica. }\end{array}$ & $\begin{array}{l}\text { FIE 31: Política de } \\
\text { prevención contra el } \\
\text { niño y la niña. }\end{array}$ & $\begin{array}{l}\text { FIE 47: Políticas sobre el } \\
\text { cuidado del medio ambiente y } \\
\text { aumento de ventas de produc- } \\
\text { tos (desperdicios) a reciclador } \\
\text { formal de la provincia. }\end{array}$ & $\begin{array}{l}\text { FIE 52: Reducir } \\
\text { sanción a infrac- } \\
\text { tor. }\end{array}$ & $\begin{array}{l}\text { FIE 61: Cursos de } \\
\text { Responsabilidad } \\
\text { Social. }\end{array}$ \\
\hline $\begin{array}{l}\text { FIE 12: Hacer } \\
\text { seguimiento para } \\
\text { cumplir el contrato } \\
\text { de limpieza. }\end{array}$ & $\begin{array}{l}\text { FIE 23: Pérdida } \\
\text { de plazas de } \\
\text { nombramientos } \\
\text { docente. }\end{array}$ & FIE 43: Riesgo País. & $\begin{array}{l}\text { FIE 44: Capacitación, desa- } \\
\text { rrollo de RR.HH. }\end{array}$ & $\begin{array}{l}\text { FIE 56: Política } \\
\text { remunerativa } \\
\text { (tercerización) }\end{array}$ & $\begin{array}{l}\text { FIE 62: Rol del } \\
\text { Consejo de Facul- } \\
\text { tad Académica y } \\
\text { del Consejo Univer- } \\
\text { sitario de la UP. }\end{array}$ \\
\hline $\begin{array}{l}\text { FIE 13: Automati- } \\
\text { zación de Oficinas } \\
\text { dependientes de la } \\
\text { Facultad Académica } \\
\text { de la UP. }\end{array}$ & $\begin{array}{l}\text { FIE 27: Muy } \\
\text { bajos los gastos } \\
\text { en formación } \\
\text { y capacitación } \\
\text { docente. }\end{array}$ & $\begin{array}{l}\text { FIE 34: Políticas } \\
\text { sobre el cuidado y } \\
\text { preservación del } \\
\text { Medio Ambiente. }\end{array}$ & $\begin{array}{l}\text { FIE 41: Normatividad (Pro- } \\
\text { veedor externo - electrónico y } \\
\text { comunicación). }\end{array}$ & $\begin{array}{l}\text { FIE 51: Reduc- } \\
\text { ción de la basura. }\end{array}$ & $\begin{array}{l}\text { FIE 63: Fortale- } \\
\text { cimiento de ética } \\
\text { y valores en los } \\
\text { recursos humanos } \\
\text { de la UP. }\end{array}$ \\
\hline $\begin{array}{l}\text { FIE 14: Seguridad en } \\
\text { la transmisión de la } \\
\text { información. }\end{array}$ & $\begin{array}{l}\text { FIE 22: Poco nivel } \\
\text { de inversiones. }\end{array}$ & & & $\begin{array}{l}\text { FIE 54: Rol de los } \\
\text { grupos de poder } \\
\text { para aprobar el } \\
\text { PEEI UP. }\end{array}$ & $\begin{array}{l}\text { FIE 64: Seguridad } \\
\text { estudiantil. }\end{array}$ \\
\hline \multirow[t]{2}{*}{$\begin{array}{l}\text { FIE 19: Implemen- } \\
\text { tación del Planea- } \\
\text { miento de Escena- } \\
\text { rios Estratégicos } \\
\text { Institucional UP. }\end{array}$} & & & & $\begin{array}{l}\text { FIE 55: Tenden- } \\
\text { cias de cambios } \\
\text { normativos. }\end{array}$ & $\begin{array}{l}\text { FIE 67: Política de } \\
\text { gasto público. }\end{array}$ \\
\hline & & & $\begin{array}{l}\text { FIE 46: Impacto en buena } \\
\text { salud mental de estudiantes } \\
\text { Facultades Académicas UP. }\end{array}$ & & \\
\hline
\end{tabular}

Fuente: Elaboración propia, 2019.

Tabla 4

Paso 4: Matriz Impacto - Incertidumbre universidad pública

\begin{tabular}{|c|c|c|c|c|}
\hline \multicolumn{3}{|c|}{ Paso 4: Matrız Impacto - Incertıdumbre unıversidad publıca } & \multirow{3}{*}{\multicolumn{2}{|c|}{ CRÍTI }} \\
\hline \multicolumn{3}{|c|}{ Grado de Incertidumbre } & & \\
\hline BAJO & MEDIO & ALTO & & \\
\hline $\begin{array}{l}\text { 41: Normatividad (proveedor exter- } \\
\text { no - electrónico y comunicaciones) }\end{array}$ & $\begin{array}{l}\text { 13: Automatización de oficinas de las } \\
\text { Facultades Académicas. I. } \\
\text { 21: Estabilidad Macroeconómica. }\end{array}$ & \multirow{5}{*}{$\begin{array}{l}\text { 22: Poco nivel de inversiones. } \\
\text { 23: Pérdida de plazas de nombramientos } \\
\text { docentes. } \\
\text { 27: Muy bajo los gastos en formación y } \\
\text { capacitación docente. } \\
\text { 54: Rol de los grupos de poder para } \\
\text { aprobar el PEEI-UP. } \\
\text { 19:Implementación del Planeamiento de } \\
\text { Escenarios Estratégicos Institucional. }\end{array}$} & \multirow{5}{*}{ ALTO } & \multirow{7}{*}{ 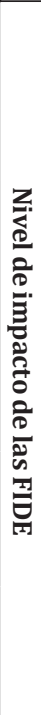 } \\
\hline 43: Riesgo País. & $\begin{array}{l}\text { 46: Impacto en buena salud mental de } \\
\text { estudiantes. }\end{array}$ & & & \\
\hline $\begin{array}{l}\text { 44: Capacitación y desarrollo de } \\
\text { RR.HH. }\end{array}$ & $\begin{array}{l}\text { 52: Reducción de sanciones de infractores. } \\
\text { 62: Rol del Consejo de Facultad Académica }\end{array}$ & & & \\
\hline $\begin{array}{l}\text { 56: Política remunerativa (terceri- } \\
\text { zación) }\end{array}$ & $\begin{array}{l}\text { 63: Fortalecimiento de ética y valores. } \\
\text { 64: Seguridad estudiantil. }\end{array}$ & & & \\
\hline 67: Política de gasto público & $\begin{array}{l}\text { 11: Infraestructura apropiada para tecno- } \\
\text { logía y software en management. }\end{array}$ & & & \\
\hline $\begin{array}{l}\text { 31: Políticas de Prevención contra el } \\
\text { Niño y la Niña. }\end{array}$ & $\begin{array}{l}\text { 47: Política sobre el cuidado del medio } \\
\text { ambiente y aumento de venta de produc- } \\
\text { tos (desperdicios) a reciclador formal de } \\
\text { la jurisdicción. }\end{array}$ & $\begin{array}{l}\text { 61: Cursos de Responsabilidad Social. } \\
\text { 55: Tendencias de cambios normativos. }\end{array}$ & MEDIO & \\
\hline $\begin{array}{l}\text { 12: Hacer seguimiento para cumplir } \\
\text { el contrato de limpieza. } \\
\text { 14: Seguridad en la transmisión de } \\
\text { la información. }\end{array}$ & \multicolumn{2}{|l|}{ 51: Reducción de la basura. } & BAJO & \\
\hline
\end{tabular}

\begin{tabular}{|c|c|c|c|c|}
\hline \multicolumn{3}{|c|}{ Grado de Incertidumbre } & & \\
\hline BAJO & MEDIO & ALTO & & \\
\hline $41,43,44,56,67$ & $13,21,46,52,62,63,64,11$ & $22,23,27,24,19$ & ALTO & $\Xi$ \\
\hline 31 & 47 & 61,55 & MEDIO & "艹 \\
\hline 12,14 & 51 & & BAJO & 5 \\
\hline
\end{tabular}

\begin{tabular}{|l|l|l|}
\hline 5 & 8 & 5 \\
\hline 1 & 1 & 2 \\
\hline 2 & 1 & 0 \\
\hline 8 & 10 & 7 \\
\hline
\end{tabular}

Fuente: Elaboración propia, 2019. 


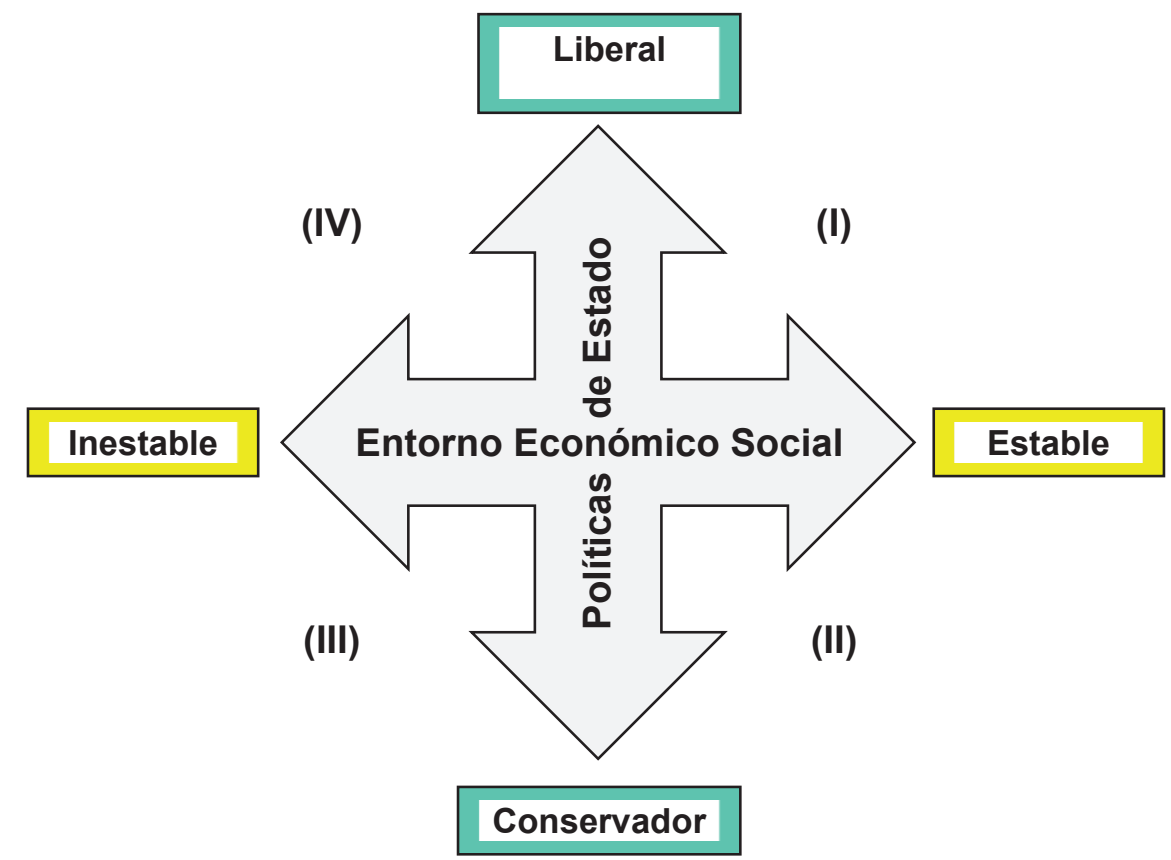

Figura 2. Paso 4: Lógica de escenarios Universidad Pública. Elaboración propia, 2019.

Tabla 5

FIE alto-alto y los ejes de incertidumbre

\begin{tabular}{|c|c|c|}
\hline 1 & 22: Poco nivel de Inversiones en las Facultades Académicas. & Entorno Económico y Social \\
\hline 2 & $\begin{array}{l}\text { 23: Pérdida de plazas de nombramiento docente otorgado por } \\
\text { el MEF - Perú. }\end{array}$ & Entorno Económico y Social. \\
\hline 3 & $\begin{array}{l}\text { 24: Alto índice de gastos no ejecutados en formación y capacita- } \\
\text { ción, desarrollo de los recursos humanos UP. }\end{array}$ & Entorno Económico y Social. \\
\hline 4 & 54: Rol del grupo político de poder. & Política de gobierno UP. \\
\hline 5 & $\begin{array}{l}\text { 61: Cursos de responsabilidad social - currículo y plan de } \\
\text { estudios UP. }\end{array}$ & Política de gobierno de las Facultades Académicas UP. \\
\hline 6 & $\begin{array}{l}\text { 66: Implementación del Planeamiento de Escenarios Estratégi- } \\
\text { cos Institucional UP. }\end{array}$ & $\begin{array}{l}\text { Política de gobierno } \\
\text { Consejo de Facultad de las Facultades Académicas y Consejo Uni- } \\
\text { versitario UP. }\end{array}$ \\
\hline 7 & 68: Tendencia de cambios normativos. & Factor Legal - Consejo Universitario; Asamblea Universitario UP. \\
\hline
\end{tabular}

Fuente: Elaboración propia, 2019.

Fase 5: Escenarios. Decisión de escenario y cuadro comparativa de escenario

Se realizó la Matriz Debilidad, Oportunidad, Fortaleza, Amenaza (FODA) del planeamiento estratégico en las universidades públicas. Véase tabla $\mathrm{N}^{\circ} 6$ y la tabla $\mathrm{N}^{\circ} 7$, matriz FODA de la universidad pública.

\section{DISCUSIÓN}

Los resultados de la investigación muestran que la Universidad Pública procurará alzar sus niveles de competencias de calidad en capacidades y recursos para mantener vigente el Licenciamiento y los estándares del mejoramiento continuo de la calidad que exige la Ley
Universitaria 30220 y la SUNEDU. Por otro lado, la Matriz FODA indica que el orden en que debería implantarse las estrategias seleccionadas es el siguiente:

1. Desarrollar el planeamiento de escenarios estratégicos institucional en la UP.

2. Establecer convenio con entidades reconocidas de revistas indexadas para difundir investigación de los docentes, estudiantes.

3. Desarrollar programas de capacitación en investigación y didáctica universitaria para los docentes. 
Planeamiento de Escenarios Estratégicos en la Universidad Pública del Perú

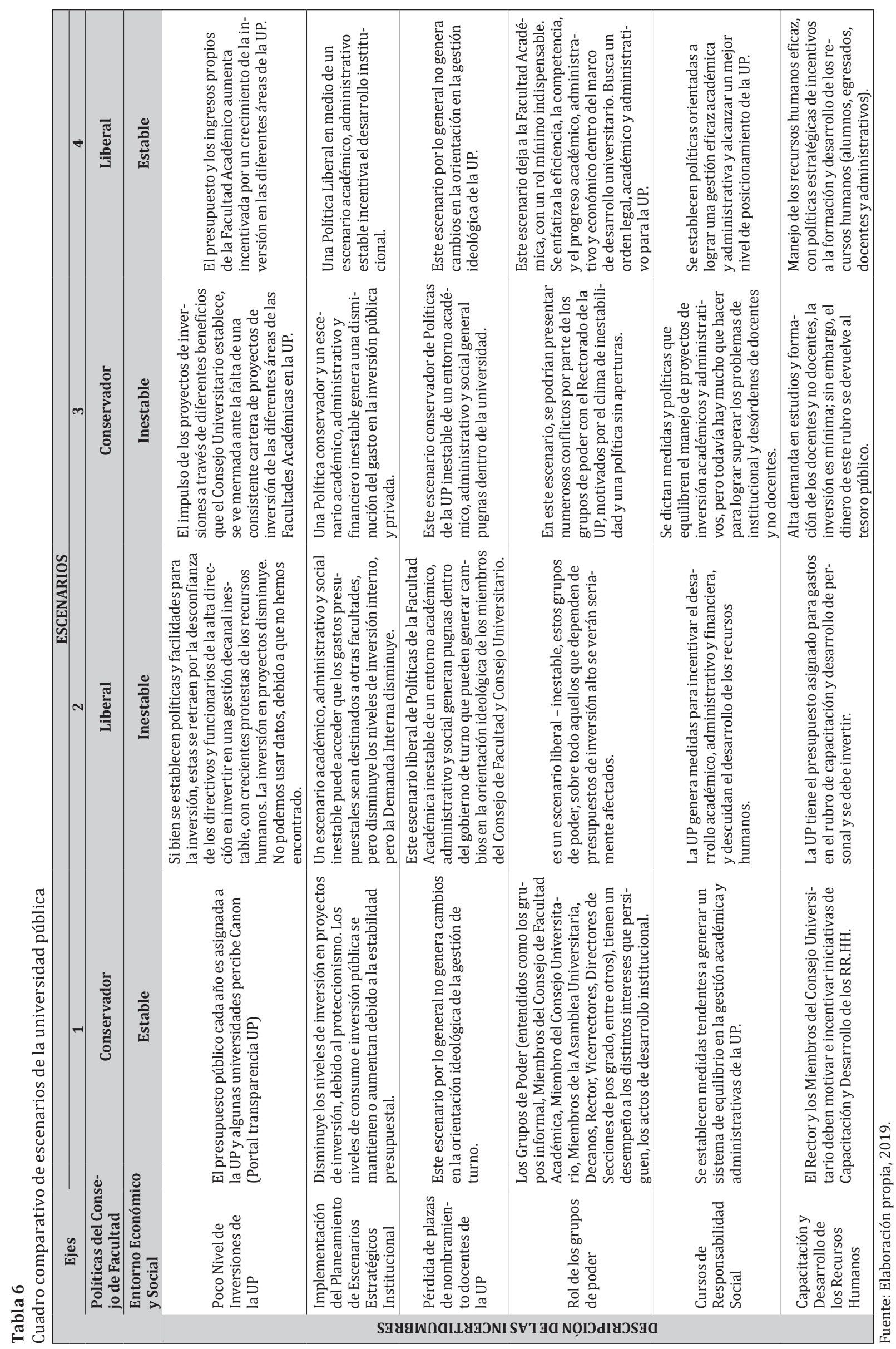


Tabla 7

Matriz FODA de la universidad pública

\begin{tabular}{|c|c|c|}
\hline & $\begin{array}{l}\text { FORTALEZAS (F). } \\
\text { 1. Acreditación por SINEACE-SUNEDU. } \\
\text { 2. Existencia de procesos de planeamiento } \\
\text { de escenarios estratégico y operativo en } \\
\text { ejecución } \\
\text { 3. Nuevos currículos de estudio } \\
\text { 4. Docentes con grado de Maestro y Doctor. } \\
\text { 5. Equipamiento de mejora de infraestructu- } \\
\text { ra, tecnología y de laboratorios } \\
\text { 6. Acceso a Internet ilimitado. }\end{array}$ & $\begin{array}{l}\text { DEBILIDADES (D). } \\
\text { 1. Alto número de Docentes mayores de } \\
65 \text { años de edad. } \\
\text { 2. Alto número de docentes sin prepara- } \\
\text { ción pedagógica y didáctica universi- } \\
\text { taria. } \\
\text { 3. Bajo nivel académico de docentes y } \\
\text { estudiantes } \\
\text { 4. Servicio al cliente } \\
\text { 5. Prestigio institucional } \\
\text { 6. Alto número de docentes que no hacen } \\
\text { investigación } \\
\text { 7. Licenciamiento-SUNEDU } \\
\text { 8. Gestión financiera }\end{array}$ \\
\hline $\begin{array}{l}\text { OPORTUNIDADES (0). } \\
\text { 1. Existencia de instituciones nacionales } \\
\text { e internacionales con las que podrían } \\
\text { establecerse convenios de cooperación. } \\
\text { 2. Demanda de proyectos productivos en } \\
\text { la jurisdicción y en el país. } \\
\text { 3. Ley Universitaria } \mathrm{N}^{\circ} 30220 \\
\text { 4. Incentivos alto a investigadores del } \\
\text { Estado. }\end{array}$ & $\begin{array}{l}\text { ESTRATEGIAS FO. } \\
\text { 1. Establecer convenio con entidades recono- } \\
\text { cidas de revistas indexadas para difundir } \\
\text { investigación de los docentes, estudiantes. } \\
\text { 2. Desarrollar proyectos de responsabilidad } \\
\text { social por docentes y estudiantes a nivel } \\
\text { nacional }\end{array}$ & $\begin{array}{l}\text { ESTRATEGIAS DO. } \\
\text { 1. Establecer estándar de selección y re- } \\
\text { tención de docentes de alto rendimiento } \\
\text { mayores de } 75 \text { años de edad. } \\
\text { 2. Desarrollar Planeamiento de escena- } \\
\text { rios estratégicos institucional en la UP. }\end{array}$ \\
\hline $\begin{array}{l}\text { AMENAZAS (A). } \\
\text { 1. Inestabilidad política debido a enfren- } \\
\text { tamiento del Poder Legislativo con el } \\
\text { Poder Ejecutivo; } \\
\text { 2. Incapacidad del Estado para satis- } \\
\text { facer necesidades de universidades } \\
\text { estatales. } \\
\text { 3. Desinterés y desconfianza de la comu- } \\
\text { nidad empresarial y del Estado en las } \\
\text { universidades estatales para generar } \\
\text { proyectos conjuntos. } \\
\text { 4. Deterioro generalizado de la moral } \\
\text { pública. } \\
\text { 5. Estancamiento de mejora del Estado en } \\
\text { el ámbito de educación }\end{array}$ & $\begin{array}{l}\text { ESTRATEGIAS FA. } \\
\text { 1. Desarrollar proyectos de formación/capa- } \\
\text { citación para MYPES, a cargo de docentes } \\
\text { y estudiantes. } \\
\text { 2. Desarrollar programas de capacitación } \\
\text { en investigación y didáctica universitaria } \\
\text { para los docentes. }\end{array}$ & $\begin{array}{l}\text { ESTRATEGIAS DA. } \\
\text { 1. Crear la Escuela de Formación Profesio- } \\
\text { nal de Gestión Pública. } \\
\text { 2. Implementar la Sección de Postgrado. } \\
\text { 3. Crear Sistema Integrado de Gestión } \\
\text { Administrativa (SIGA) }\end{array}$ \\
\hline
\end{tabular}

Fuente: Elaboración propia, 2019.

4. Establecer estándar de selección y retención de docentes de alto rendimiento mayores de 75 años.

5. Desarrollar proyectos de responsabilidad social por docentes y estudiantes a nivel nacional

6. Crear la Escuela de Formación Profesional de Gestión Pública.

\section{CONCLUSIONES}

Concluimos que la Universidad Pública en el Perú está regulada por la Ley Universitaria $\mathrm{N}^{\circ}$ 30220 y la SUNEDU para mantener vigente el Licenciamiento y los estándares del mejoramiento continuo de la calidad; finalmente, debe poner énfasis en desarrollar las acciones estratégicas resultado de la investigación a través de la matriz FODA en orden de prioridad, de la manera siguiente:

1. Desarrollar el planeamiento de escenarios estratégicos institucional en la universidad pública.

2. Establecer convenio con entidades reconocidas de revistas indexadas para difundir investigación de los docentes, estudiantes.

3. Establecer estándar de selección y retención de docentes de alto rendimiento mayores de 75 años.

4. Desarrollar proyectos de responsabilidad social por docentes y estudiantes a nivel nacional.

5. Crear la Escuela de Formación Profesional de Gestión Pública. 
REFERENCIAS BIBLIOGRÁFICAS.

Baena P. (2015). "Planeación prospectiva estratégica". Universidad Nacional Autónomo de México - México.

Centro Nacional de Planeamiento Estratégico (Ceplan). (2017). "Resolución de Presidencia de Consejo Directivo N062-17. Guía para el planeamiento institucional".

Congreso de la República. (2014). "Ley N³0220, Ley Universitaria”. En: sunedu,gob.pe. [PDF]. 09 de julio de 2014. Fecha de consulta: 10/05/2015. Disponible en: www.sunedu.gob.pe/nueva ley universitaria 30220-2014. PDF.

Minztberg, H. (1994). On strategic planning. New York: Editorial Staff For Infoline.

Narciso, K. (2008). "Planeamiento Estratégico de la FCA-UNAC". Lima, INIFCA de la Universidad Nacional del Callao. Informe Final de Investigación.

Narciso; K. (2017). "Plan estratégico de la Facultad de Ciencias Administrativas UNMSM 2017-
2019". En: Gestión en el tercer milenio. Vol. 19-1, º37. [En línea]. Fecha de consulta: 12 de mayo 2017. Disponible en: https://revistasinvestigacion.unmsm.edu.pe/index.php/administrativas/issue/view/1272.

Porter, M. (2004). Ventaja competitiva. México DF.: Editorial Continental, S.A. de C.V. de México.

Schwatz, P. (2003). Inevitable Surprise: thinking ahead in a time of turbulence. Hudson; Gothen Books.

Superintendencia Nacional de Educación Superior Universitaria (Sunedu). (2019) "Resolución Consejo Directivo $N^{\circ} 011$ ". Plan estratégico institucional-Sunedu.

Yori, L.; Hernández de Velazco, J., y Chumaceiro, A. (2011). "Planificación de escenarios: una herramienta estratégica para el análisis del entorno". En: Revista Venezolana de Gerencia. N54, pp: $280,281,286$. 
